scan for detection of cardiac and other defects prenatally. Significant numbers of chromosomal and extra-cardiac defects in this study emphasise the importance of thorough evaluation of any fetus identified with a cardiac defect.

\section{PF.27 THE IMPACT OF THE NHS FETAL ANOMALY SCREENING PROGRAMME 2010 ON REFERRALS TO FETAL MEDICINE FOR VENTRICULOMEGALY}

doi:10.1136/archdischild-2013-303966.039

${ }^{1} \mathrm{CL}$ Yates, ${ }^{2} \mathrm{G}$ Masson. ${ }^{1}$ Keele University Medical School, Keele, UK; ${ }^{2}$ University Hospital of North Staffordshire, Newcastle, UK

The NHS Fetal Anomaly Screening Programme (FASP) is a national screening guideline for the United Kingdom to detect fetal anomalies (1). In 2010 the national guidance was updated to include measurement of the ventricular atrium, among other alterations, to screen for ventriculomegaly. The guidance states if the atrium measurement was greater than $10 \mathrm{~mm}$ this should be reported and the patient referred for further assessment and managed as for a suspected fetal anomaly (2). This change was implemented at the University Hospital of North Staffordshire on $1^{\text {st }}$ April 2011. For the purpose of this audit all cases of ventriculomegaly, including borderline measurements, detected on ultrasound scanning at UHNS within the given time periods were included. The time periods used were between March 2010 - March 2011, before the introduction of screening for ventriculomegaly (Pre-FASP), and between June 2011 - June 2012 which was after the introduction (Post-FASP). Since the change it became apparent there has been an increased demand on fetal medicine services. The audit demonstrates a fifty per cent increase in referrals to the fetal medicine team for ventriculomegaly. Issues identified from the audit included an increased number of ventriculomegaly diagnoses, a greater demand on fetal medicine services such as additional investigations and extra ultrasound scans as well as a difference in pregnancy outcomes.

\section{REFERENCES}

1. NHS Fetal Anomaly Screening Programme (NHS FASP). (2010). About the NHS Fetal Anomaly Screening Programme. Available: http://fetalanomaly.screening.nhs. uk/fetalanomalyresource/whats-in-the-hexagons 1 /about-the-nhs-fetal-anomalyscreening-programme. Last accessed 28 November 2012.

2. NHS Fetal Anomaly Screening Programme (NHS FASP). (2010). Normal variant screening (formerly known as soft markers). Available: http://fetalanomaly.screening.nhs.uk/fetalanomalyresource/whats-in-the-hexagons1/about-the-scan/normal-variant-screening. Last accessed 28 November 2012.

\section{PF.28 SPONTANEOUS RESOLUTION OF PRENATALLY DETECTED DURAL SINUS THROMBOSIS: CASE SERIES}

doi:10.1136/archdischild-2013-303966.040

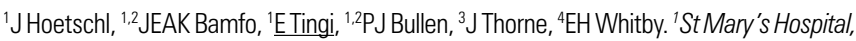
Central Manchester University Hospital NHS Foundation Trust, Manchester, UK; ${ }^{2}$ Fetal Medicine Unit, St Mary's Hospital, Central Manchester University Hospital NHS Foundation Trust, Manchester, UK; ${ }^{3}$ Department of Paediatric Neurology, Central Manchester University Hospital NHS Foundation Trust, Manchester, UK; ${ }^{4}$ Academic unit of Reproductive and developmental medicine, Jessop Wing, University of Sheffield, Sheffield, UK

Dural sinus thrombosis (DST) is a rare fetal cerebral abnormality. We present 6 cases of DST detected prenatally with ultrasound and magnetic resonance imaging (MRI). In 4 cases, the DST spontaneously resolved with good longterm neonatal outcome. Two patients chose to terminate their pregnancies.

The women presented with ultrasound findings of posterior fossa abnormalities or cysts between 20 and 27 weeks gestation. On MRI, the DST was seen as a lesion of low signal intensity with a centrally placed higher signal lesion consistent with a thrombus. The lesion was often continuous with the saggital sinus and transverse sinus with displacement of the cerebellum and occipital horns of the cerebral hemispheres. The remaining brain appeared normal. In all cases, MRI captured the spontaneous resolution.

There is a lack of information on longterm prognosis because of the small numbers of cases reported. In many cases, parents opt for termination of pregnancy because of the uncertainty about the longterm prognosis.

In conclusion, from our case series and review of the literature, it appears that spontaneous resolution of DST in the antenatal period may suggest a good long term neonatal prognosis. Where the thrombus remains extensive, prognosis should be guarded. MRI is useful for delineating resolution or regression of the thrombus. MRI can be an essential adjunct to ultrasound in the diagnosis and management of DST.

\section{PF.29 SHOULD WE RECONSIDER THE ELECTIVE MODE OF DELIVERY IN GASTROSCHISIS?}

doi:10.1136/archdischild-2013-303966.041

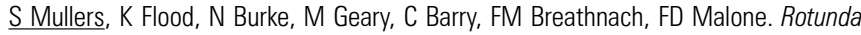
Hospital, Dublin, Ireland

The recommended mode of delivery for the fetus with an abdominal wall defect is controversial, with no evidence to support caesarean delivery other than for routine obstetric indications. We sought to review mode of delivery in cases of prenatally diagnosed gastrochisis in our centre.

This was a retrospective cohort study of prenatally diagnosed fetal gastroschisis cases in the Rotunda Hospital over a fourteen year period. Cases were identified from the Fetal Assessment Unit database.

We identified 35 cases fetal gastroschisis cases during the study period. The average age of mothers was 22.5 years. The median gestation at delivery was $35+4$ with an average birthweight of $1.97 \mathrm{~kg}$.

An elective caesarean section was performed in $13.3 \%(n=4)$ cases. Vaginal delivery was the intended mode of delivery for the remaining cases $(n=26)$ however $54 \%$ resulted in an emergency caesarean delivery with nonreassuring CTG cited as the most common indication $(64 \%, 9 / 14)$. Of these, $50 \%(7 / 14)$ were performed outside of normal working hours.

Although the numbers in our cohort are relatively small, we found a significantly high rate of caearean delivery in young women with pregnancies complicated by gastroschisis. The high proportion of cases with nonreassuring fetal testing during labour resulted in a higher than expected number of emergency deliveries which were performed outside normal working hours. Our findings suggest that re-evaluation of the optimal mode of delivery in this cohort may be warranted.

\section{PF.30 THE USE OF MICROARRAY TECHNOLOGY IN PRENATAL TESTING, PATIENT AND PARTNERS REFLECTIONS}

doi:10.1136/archdischild-2013-303966.042

${ }^{1}$ SC Hillman, ${ }^{3} \mathrm{~J}$ Skelton, ${ }^{2} \mathrm{E}$ Quinlan Jones, 'A Wilson, ${ }^{1,2} \mathrm{MD}$ Kilby. ${ }^{1}$ School of Clinical and Experimental Medicine, College of Medical and dental Sciences University of Birmingham, Birmingham, UK; '2Birmingham Women's Foundation Trust, Birmingham, UK; ${ }^{3}$ School of Health and Population Sciences, College of Medical and Dental Sciences, University of Birmingham, Birmingham, UK

In the prenatal setting, women are offered fetal karyotyping for reasons including an abnormality on ultrasound scan. Full, conventional karyotyping has been available since the 1960s but is being superseded by chromosomal microarray testing (CMA). Often the results obtained from CMA give women and their partners more information on which to make decisions and prepare. However in some cases the results can be complex and even uncertain. It is vital that we seek 
to understand the effect this new technology (and the uncertainty it can create) is having on couples. Previous qualitative work in this field is limited to women's experience of prenatal genetic testing or women's experience of having an abnormal ultrasound scan.

We present qualitative research from 25 semi-structured interviews with women (and their partners) whose babies have fetal anomalies after CMA testing. Data was analysed using framework analysis. A thematic framework was then identified by recognising emerging themes. Five themes were identified; diagnosis, genetic testing, family and support, reflections of the treatment received and emotions.

Our results show that women recall being told about common trisomies but often no further testing. Women expected the conventional karyotyping and CMA result would be normal following a normal OFPCR result. There were frequent misconceptions by couples regarding aspects of counselling/testing. Communication of variants of unknown (clinical) significance (VOUS) presents a particularly difficult challenge. Good clear communication by health care professionals is paramount. Couples should have literature to take home summarising scan anomalies and reinforcing information about genetic testing.

\section{PF.31 VITAMIN B12 DEFICIENCY AND RECURRENT PREGNANCY LOSS: IS THERE AN ASSOCIATION?}

doi:10.1136/archdischild-2013-303966.043

NB Bozreiba, SC Cooley, SCS Coulter Smith. Royal college of physician Ireland, Dublin, Ireland

Introduction The importance of preconceptual folic acid has been established. However the metabolism of folate and Vitamin B12 is interlinked. Weak association has been described between Vitamin B12 deficiency and recurrent pregnancy loss. This has led to the implementation in some centres of routine B12 levels in patients with recurrent pregnancy loss. Our goal is to determine the value of such testing and its role in the management of these cases.

Aim To determine whether the incidence of folate and/or Vitamin B12 deficiency is higher in patients with recurrent pregnancy loss than the reported national incidence of deficiency.

Methods Retrospective review of all new referrals to the recurrent miscarriage clinic at the Rotunda Hospital Dublin. Vitamin B12 and folate levels were assessed at the initial consultation.

Results National B12 levels and folate levels were accepted as $6 \%$ based on new nutritional data. We evaluated the folate and cobalamin status in 98 non-pregnant women with a history of recurrent spontaneous abortion (three or more consecutive) of unknown aetiology. Low Vitamin B12 was defined as serum value less than $190 \mathrm{ng} / \mathrm{l}$, and serum folate values less than $4.5 \mathrm{ug} / \mathrm{l}$ were considered deficient.

In total $7.1 \% \mathrm{n}(\mathrm{n}=7)$ patients were Vitamin B12 deficient and only one patient was folate deficient. This compares with a national incidence of $6 \%$. These levels were not statistically different.

Conclusion Serum concentrations of folic acid and Vitamin B12 are not significantly altered in women with unexplained recurrent miscarriage and we propose that routine testing is not warranted.

\section{PF.32 PARTIAL URORECTAL SEPTUM MALFORMATION (PURSM) SEQUENCE IN ENGLAND AND WALES: PREVALENCE, ADDITIONAL ANOMALIES, AND PREGNANCY OUTCOME}

doi:10.1136/archdischild-2013-303966.044

'PWG Tennant, 'SV Glinianaia, ${ }^{2} \mathrm{D}$ Wellesley, ${ }^{1,3} \mathrm{~J}$ Rankin. ${ }^{1}$ Institute of Health \& Society, Newcastle University, Newcastle-upon-Tyne, UK; ' Wessex Clinical Genetics Service, Princess Anne Hospital, Southampton, UK; ${ }^{3}$ Regional Maternity Survey Office, Newcastle-upon-Tyne, UK

Background Partial urorectal septum malformation (pURSM) sequence (or 'persistent cloaca') is a rare congenital anomaly characterised by a confluence of the urethral, anal, and genital openings. This study describes the prevalence, additional anomalies, and pregnancy outcomes of pURSM sequence in England and Wales.

Methods All cases of pURSM sequence notified to seven congenital anomaly registers in England and Wales during 1985-2010, whether delivered as live births, spontaneous fetal deaths ( $\geq 20$ weeks gestation), or elective terminations of pregnancy for fetal anomaly (any gestation), formed this population-based case series. Risks of spontaneous fetal and infant death were examined by the Kaplan-Meier method. Differences in prevalence over time, and between regions, were examined by multilevel Poisson regression.

Results 117 cases were recorded among 4,251,241 total births. 58 $(50 \%)$ were delivered as live births, $6(5 \%)$ as spontaneous fetal deaths, and $53(45 \%)$ as elective terminations. The total prevalence was 2.8 (95\% CI: 2.3-3.4) per 100,000 total births, increasing significantly over time $(p=0.002)$ and differing significantly between regions $(p=0.005) .77$ cases $(66 \%)$ had at least one additional major congenital anomaly outside the perineum, including 67 (57\%) with renal, $29(25 \%)$ with musculoskeletal, $26(23 \%)$ with digestive system, and 24 (21\%) with cardiovascular anomalies. The risks of spontaneous fetal and infant death were estimated as 8.9\% (95\% CI: $4.1-18.8)$ and $26.3 \%$ (95\% CI: $15.1-43.4)$ respectively.

Conclusions This is the largest study of the epidemiology of pURSM sequence. This information should be valuable for families and health professionals whenever a case of pURSM sequence is diagnosed.

\section{PF.33 WITHDRAWN BY AUTHOR}

\section{PF.34 EFFECT OF ANTIHYPERTENSIVE THERAPY ON UTERINE ARTERY AND FETAL DOPPLERS: SYSTEMATIC REVIEW AND META-ANALYSIS}

doi:10.1136/archdischild-2013-303966.045

${ }^{1} \mathrm{~N}$ Suff, ${ }^{2} \mathrm{D}$ Cooper, ${ }^{3} \mathrm{~A}$ Khalil. ${ }^{1} / n$ situte of Women's Health, University College London, London, UK; ${ }^{2}$ Fetal Medicine Unit, St. George's University Hospital, London, UK; ${ }^{3 K i n g}$ 's College London, London, UK

Introduction Antihypertensive drugs lower blood pressure by direct vascular effects or central vasodilatory mechanisms. Methyldopa is a commonly used in hypertensive disorders in pregnancy. Although methyldopa is effective in controlling maternal blood pressure, its effects on fetal growth and wellbeing is unknown. The aim of this study was to ascertain the effect of methyldopa on uterine artery and fetal dopplers in women with hypertensive disorders in pregnancy.

Methods MEDLINE (1966 - March 2012), EMBASE (1974 - March 2012) and The Cochrane library (inception-March 2012), utilising combinations of the terms methyldopa, hypertens*, pre-eclamp*, preeclamp*, pre eclamp*, pregnan*, uterine, uteroplacental, umbilical, middle cerebral artery and doppler. Between-study heterogeneity was assessed using the $\mathrm{I}^{2}$ statistic.

Results The search yielded 429 citations. Full manuscripts were retrieved for 32 and 6 were included in the review and meta-analysis. Six studies $(n=188)$ have reported the effect of methyldopa on uterine artery doppler pulsatility index (PI). The standardised mean difference (SMD) was -0.22 (95\% CI: -0.43 to -0.02$)$. Five studies $(\mathrm{n}=110)$ were included that evaluated the effect of methyldopa on umbilical artery Doppler PI. The SMD was -0.05 (95\% CI: -0.32 to $0.21)$. Four studies $(n=82)$ reported the effect of methyldopa on middle cerebral artery PI. The SMD was -0.34 ( $95 \%$ CI: -0.65 to 0.03 ).

Conclusion In pregnancies complicated by hypertension, methyldopa seems to have a small favourable effect on uterine artery dopplers. These results provide reassurance that the maternal hypotensive effect of methyldopa is unlikely to have a clinically significant adverse effect on the pregnancy. 